\title{
Decadal trends in the diurnal variation of galactic cosmic rays observed using neutron monitor data
}

\author{
Simon Thomas ${ }^{1,2}$, Mathew Owens ${ }^{1}$, Mike Lockwood ${ }^{1}$, and Chris Owen ${ }^{2}$ \\ ${ }^{1}$ Department of Meteorology, University of Reading, Earley Gate, Whiteknights, RG6 6BB, Reading, UK \\ ${ }^{2}$ Mullard Space Science Laboratory, University College London, Holmbury St. Mary, RH5 6NT, Dorking, Surrey, UK \\ Correspondence to: Simon Thomas (s.r.thomas@ucl.ac.uk)
}

Received: 4 November 2016 - Revised: 30 May 2017 - Accepted: 12 June 2017 - Published: 17 July 2017

\begin{abstract}
The diurnal variation (DV) in galactic cosmic ray (GCR) flux is a widely observed phenomenon in neutron monitor data. The background variation considered primarily in this study is due to the balance between the convection of energetic particles away from the Sun and the inward diffusion of energetic particles along magnetic field lines. However, there are also times of enhanced DV following geomagnetic disturbances caused by coronal mass ejections or corotating interaction regions. In this study we investigate changes in the DV over four solar cycles using ground-based neutron monitors at different magnetic latitudes and longitudes at Earth. We divide all of the hourly neutron monitor data into magnetic polarity cycles to investigate cycleto-cycle variations in the phase and amplitude of the DV. The results show, in general, a similarity between each of the $A<0$ cycles and $A>0$ cycles, but with a phase change between the two. To investigate this further, we split the neutron monitor data by solar magnetic polarity between times when the dominant polarity was either directed outward (positive) or inward (negative) at the northern solar pole. We find that the maxima and minima of the DV changes by, typically, $1-2 \mathrm{~h}$ between the two polarity states for all non-polar neutron monitors. This difference between cycles becomes even larger in amplitude and phase with the removal of periods with enhanced DV caused by solar wind transients. The time difference between polarity cycles is found to vary in a 22-year cycle for both the maximum and minimum times of the DV. The times of the maximum and minimum in the DV do not always vary in the same manner between $A>0$ and $A<0$ polarity cycles, suggesting a slight change in the anisotropy vector of GCRs arriving at Earth between polarity cycles. Polar neutron monitors show differences in phase between polarity cycles which have asymptotic directions at
\end{abstract}

mid-to-high latitudes. All neutron monitors show changes in the amplitude of the DV with solar polarity, with the amplitude of the DV being a factor of 2 greater in $A<0$ cycles than $A>0$ cycles. In most cases the change in timing of the maximum /minimum is greatest with the stations' geomagnetic cut-off rigidity shows little variation in the DV phase with latitude. We conclude that the change in the DV with the dominant solar polar polarity is not as simple as a phase change, but rather an asymmetric variation which is sensitive to the neutron monitor's asymptotic viewing direction.

Keywords. Interplanetary physics (cosmic rays)

\section{Introduction}

Galactic cosmic rays (GCRs) are high-energy (typically classified as $>100 \mathrm{MeV}$ ) ions (predominantly protons) that originate from galactic sources such as supernovae. When they arrive at Earth, they collide with atmospheric molecules and produce a shower of secondary particles which cascade down to the surface (e.g. Bazilevskaya et al., 2008). Neutrons and protons are examples of secondary species that can be detected at the surface. Across the globe, there is a network of neutron monitors (NMs), some of which have been in operation from the 1950s (e.g. Moraal et al., 2000). NM count rates act as an excellent proxy for the flux of GCRs incident on Earth's atmosphere (e.g. Mishev et al., 2013; Vainio et al., 2009; Usoskin et al., 2011). The dependence of NM count rates on meteorological parameters, such as atmospheric pressure and thickness, means that they need correction (Raubenheimer and Stoker, 1974). Such a correction is routinely applied as part of the data calibration of all NMs used in this study. 
GCRs detected by a NM are unlikely to have precipitated vertically downward but rather will have been deflected by the Earth's magnetosphere. The geomagnetic cut-off rigidity of a particular NM is influenced by the structure of the magnetosphere. Near the equator, the geomagnetic cut-off rigidity is far greater than at the poles due to the configuration of the magnetosphere and the energy that the particles required to cross the closed magnetic field lines near the equator. Smart et al. (1965) produced an analytical model to trace GCR trajectories back from the origin of the GCR cascade (a height of 20-25 km in the atmosphere), through the magnetosphere to show the potential range of paths of GCRs of different energies arriving at a particular NM. The collection of potential trajectories that different energy GCRs can take to a particular NM maps out a 3-D swathe of the sky known as the monitor's "asymptotic direction" (AD; Plainaki et al., 2009). However, the AD of a given NM can be varied on short timescales as solar wind transients can distort the magnetosphere (Flückiger et al., 1985). Reconnection processes (Dungey, 1961) associated with solar wind transients and other minor alterations to the magnetic field (Milan et al., 2012) can happen at any moment and have limited predictability (e.g. Owens and Cargill, 2004; Thomas et al., 2016), thus making the deduction of a neutron monitor's AD at any given time difficult.

NM count rates are also influenced directly by heliospheric conditions (e.g. Jokipii et al., 1977; Thomas et al., 2014b). On annual timescales, GCRs are modulated by solar variability due to changes in the intensity and variability of the heliospheric magnetic field (HMF) via particle drifts, diffusion, convection with the solar wind and adiabatic deceleration (e.g. Parker, 1965; Gil et al., 2015). Thus, GCR flux at Earth is changed by both the sense of the Sun's magnetic field polarity via particle drifts (Jokipii et al., 1977) and changes in the HMF via effective "shielding" (e.g. Rouillard and Lockwood, 2004; Thomas et al., 2014a). The Sun's polarity is classified by the dominant solar polar polarity, $A$, being outwards in the northern pole (denoted $A>0$ ) or inwards (denoted $A<0$ ) for this purpose (e.g. Ahluwalia, 1994; Potgieter, 2013). Due to the differences in drift patterns and the HMF field on these long timescales, it is reasonable to expect that a change in the preferred direction of anisotropy on shorter timescales between the two polarities is possible.

On hourly timescales, variations in the HMF strength related to solar wind transients (such as coronal mass ejections) can result in rapid changes in GCR flux at Earth, known as Forbush decreases (Forbush, 1937). Indeed, even remote coronal mass ejections can have important effects on shortterm GCR flux (e.g. Thomas et al., 2015; Dumbovic et al., 2015), implying that for GCR modulation at Earth to be fully understood, knowledge of structures in the magnetic field of the whole heliosphere is required. Particle drift effects are also evident when considering other heliospheric transients. Boundaries between fast and slow solar wind, commonly known as corotating interaction regions (CIRs), mod- ulate NM count rates when crossing Earth (Richardson et al., 1996). CIRs are often co-located with the heliospheric current sheet (HCS), dividing opposing magnetic polarities. Thomas et al. (2014b) found that the compression effects of stream-stream interactions within the HCS cause a reduction in GCR flux at Earth, but that even without this compression, the HCS can significantly influence NM count rates. The implication of this result is that particle drifts should be considered a key modulating parameter for GCR modulation.

A diurnal variation (DV) of neutron monitor counts at any one station has long been observed and attributed to an anisotropy in GCRs reaching the Earth (e.g. Forbush and Venkatesan, 1960; Lapointe and Rose, 1961; Rao et al., 1963; Parker, 1964). This anisotropy is thought to arise due to the balance between the convection of GCRs away from the Sun and the inward diffusion of GCRs (Mishra and Mishra, 2006). A complete knowledge of the HMF is important for understanding this variation as small-scale features in the heliosphere can have a significant effect on the DV phase and amplitude (Bieber and Evenson, 1998). This is particularly true as the background DV is known to be particularly prevalent during non-disturbed solar wind conditions, when there are no other solar wind transients present. On average, the DV has an amplitude of around $0.5 \%$. The amplitude can be larger during disturbed heliospheric conditions when the DV is influenced by the presence of solar wind transients. This is primarily observed in NM data during the recovery phase of Forbush decreases caused by coronal mass ejections (e.g. Tezari and Mavromichalaki, 2016) and are named DV "trains". These DV trains can cause DV amplitudes of up to $5 \%$ which can be out of phase with the background DV, as they are caused by the modulating capability of solar wind transients. Therefore, when studying the DV in GCR flux, it is important to try to distinguish between these two variations.

The component of the DV that is associated with meteorological changes, such as changes in pressure, temperature, humidity and atmospheric density, are effectively removed in all NM data (e.g. Bercovitch and Robertson, 1965; Raubenheimer and Stoker, 1974; Kobelev et al., 2011). However, the average DV is generally regarded as inconsistent with what may be expected from meteorological effects (Forbush, 1973), which should be strongly ordered by local time. Initial testing of the mean DV's amplitude and phase difference with season showed no significant differences meaning that an atmospheric cause for our results is unlikely.

Several authors (e.g. Forbush, 1969; Bieber and Chen, 1991) have studied phase changes in the DV and interpreted them in terms of the anisotropy in GCRs reaching Earth. Using NMs and ionisation chamber data from 1937 to 1967, Forbush (1969) found a change in the time of the maximum in GCR flux between three solar cycles with a period of two cycles. Bieber and Chen (1991) then extended this analysis to three further cycles and showed that there was indeed a 22-year cycle in the DV maxima. Oh et al. (2010) used fur- 
ther statistical analyses to show that the phase of the diurnal cycle was actually a 22-year and 11-year variation superimposed and suggested the roots of these are particle drifts and the HMF strength respectively. This study updates the work to include the late 1980s and also shows a solar cycle periodicity in the amplitude of the DV. We include neutron monitors from 1955 to 2014 for a larger selection of different latitudes and longitudes and present the data in a new manner to compare local changes in the DV phase.

In this study, we use a large selection of long-running neutron monitor stations, using the criteria that all NMs that we use in this study must have been running for a period covering four solar cycles. The NMs that we have selected are listed in Table 1: Alma-Ata B (Kazakhstan), Climax (USA), Deep River (Canada), Hermanus (South Africa), Kiel (Germany), McMurdo (Antarctica), Newark (USA), Oulu (Finland), South Pole (Antarctica) and Thule (Greenland). Note that not all NMs are still in use, for example Climax, but all have at least 38 years of data available. Geographic latitudes and longitudes are also listed in the table. These are accessed from the neutron monitor database (NMDB; Mavromichalaki et al., 2011), with the exception of the Climax and Deep River neutron monitors, where data were accessed from the Izmiran database of closed long-term neutron monitor data, and Hermanus, accessed from the North-West University website (Moraal and Stoker, 2010). Note that Alma-Ata B (herein just called Alma-Ata), Climax and South Pole are all high-altitude NMs.

The geomagnetic cut-off rigidities can be found in the final column of Table 1 and are each taken from the NMDB database at http://www.nmdb.eu. The cut-off rigidity is defined as the rigidity required for a GCR to overcome the geomagnetic field and to reach the surface at a given location. Thus, polar neutron monitors will have a small geomagnetic cut-off rigidity as GCRs can arrive more readily along the open magnetic field lines in these regions than the closed field line around the equator, where the cut-off is considerably higher. Here, and as seen in Table 1, we are using a wide sample of a range of geomagnetic latitudes, including polar stations with very low geomagnetic cut-off rigidities (e.g. $0.10 \mathrm{GV}$ at the South Pole) and stations with relatively high cut-off rigidities (e.g. $6.69 \mathrm{GV}$ at Alma-Ata). It is worth noting that the geomagnetic cut-off rigidities of the NMs change with time (Herbst et al., 2013). This is particularly obvious for Newark and Thule, which were on similar longitudes to the magnetic north pole at the start of the NM records and Hermanus who's geomagnetic cut-off rigidity has been reduced with time with the development of the South Atlantic anomaly (Cordaro et al., 2016). Although these changes could influence our analysis of the DV, the changes are accounted for with the length of the data we use and the associated error bars. In the next section we shall investigate the DV at each NM and how it changes from cycle to cycle.

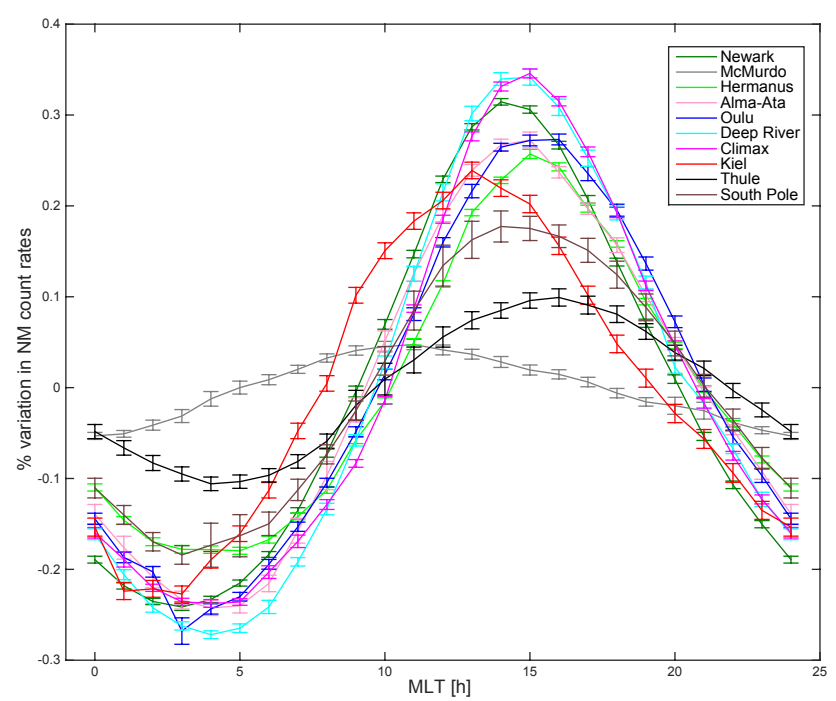

Figure 1. Percentage change in neutron monitor counts with respect to a 10-day running mean, averaged for each hour of the day (in MLT) for all available days of data for each neutron monitor. The colours represent different neutron monitors given by the key in the top-right of the figure. The error bars are the standard error on the means.

\section{Mean diurnal variations from the neutron monitor network}

We now investigate the mean DV from each NM selected from the global network, as listed in Table 1. For the majority of NMs, a smooth curve in count rate has previously been observed (Forbush, 1969) with a maximum and minimum at distinct local times. By converting each NM from coordinated universal time (UTC) to magnetic local time (MLT), the times of the maxima and minima in the variation should align as all monitors will be pointing in the same direction into space at each given time (with some variance due to the slightly different shapes of the ADs of the various NMs associated with the structure of the geomagnetic field). On average, the maxima in the DV in previous literature has been found to occur between 15:00 and 18:00 MLT (e.g. Pomerantz and Duggal, 1970).

Figure 1 shows the percentage change in hourly NM count rates with respect to a 10-day running mean, used to remove the effects of long-term solar variability but to average out the DV from the mean. For each hourly data point, a 10-day running mean is computed around it. The running mean is then subtracted from the data point and then the resulting difference is divided by the running mean and multiplied by 100 to gain a percentage. The mean of all the available data for each hour of the day is then taken and plotted in Fig. 1 against the station's MLT. The error bars represent the standard error on the mean for each hourly value, which is small given the large amount of data represented by each data point. Each coloured line displayed in Fig. 1 represents a different NM, 
Table 1. Neutron monitors used in the study with their dates of use, latitudes, longitudes and cut-off rigidities. Note that South Pole does not have a particular latitude due to its polar location. Table collated from data collected using the neutron monitor database at http:// www.nmdb.eu apart from Climax and Deep River from http://cr0.izmiran.ru/common/links.htm and Hermanus from http://www.nwu.ac.za/ neutron-monitor-data. Note that Alma-Ata B, Climax and South Pole are all located at high altitudes.

\begin{tabular}{|c|c|c|c|c|c|}
\hline $\begin{array}{l}\text { Neutron } \\
\text { monitor }\end{array}$ & $\begin{array}{l}\text { Start } \\
\text { year }\end{array}$ & $\begin{array}{l}\text { End } \\
\text { year }\end{array}$ & $\begin{array}{l}\text { Latitude } \\
\left({ }^{\circ}\right)\end{array}$ & $\begin{array}{l}\text { Longitude } \\
\left({ }^{\circ}\right)\end{array}$ & $\begin{array}{r}\text { Rigidity } \\
\text { cut-off }(\mathrm{GV})\end{array}$ \\
\hline Alma-Ata B & 1973 & Present & 43.1 north & 76.6 east & 6.69 \\
\hline Climax & 1953 & 2007 & 37.4 north & 106.2 west & 2.92 \\
\hline Deep River & 1957 & 1995 & 46.1 north & 77.5 east & 1.02 \\
\hline Hermanus & 1957 & Present & 34.4 south & 19.2 east & 4.90 \\
\hline Kiel & 1964 & Present & 54.3 north & 10.1 east & 2.36 \\
\hline McMurdo & 1960 & Present & 77.9 south & 166.6 east & 0.30 \\
\hline Newark & 1964 & Present & 37.9 north & 75.8 west & 2.40 \\
\hline Oulu & 1964 & Present & 65.1 north & 25.5 east & 0.80 \\
\hline South Pole & 1964 & Present & 90.0 south & 0 & 0.10 \\
\hline Thule & 1957 & Present & 76.5 north & 68.7 west & 0.30 \\
\hline
\end{tabular}

showing all the NMs listed in Table 1 and used throughout this study.

In agreement with previous studies, we find that most NMs have a peak in the variation at 14:00-17:00 MLT, which falls close to the range found by Pomerantz and Duggal (1970) of 15:00-18:00 MLT. The AD of a given NM is often offset in a complex manner between each monitor, thus each NM will be sampling a different region of the sky at the same MLT. The larger phase difference for McMurdo (as seen by the grey line in Fig. 1, peaking at an MLT of $10 \mathrm{~h}$ ) is related to the low amplitude of the variation and is due to its location at a very high latitude. The ADs of most NMs are shifted eastwards by approximately $60^{\circ}$, whereas McMurdo's AD shifts differently resulting in a different phase in the DV. The $\mathrm{AD}$ for McMurdo also lies in mid-high latitude in the Southern Hemisphere and samples GCRs arriving from a comparatively narrow range of the sky with a small latitudinal span. South Pole NM does not have the same effect as McMurdo $\mathrm{NM}$ as it is somewhat removed from the southern magnetic pole, with a less unusual AD.

\section{Are there phase changes in the diurnal variation?}

In this section, we investigate changes in the phase and amplitude of the DV with heliospheric polarity. Bieber and Chen (1991) found a link between these phenomena and here we update and expand upon their analysis, including data from two further solar cycles and a broader range of magnetic latitudes and longitudes. We first separate the NM data for polarity cycle, as defined in Thomas et al. (2014a). For each NM, this gives at least four polarity cycles worth of mean DVs to compare. The mean DVs for four polarity cycles (from the period 1970-2014) are shown in Fig. 2. The examples shown are for the Hermanus, Newark, Alma-Ata and McMurdo neutron monitors in MLT, each chosen to provide a broad range of magnetic latitudes and longitudes. For exam- ple, Hermanus is located in the Southern Hemisphere and is one of the longest running NMs, whereas Newark and AlmaAta are on opposing sides of the Northern Hemisphere in terms of longitude. McMurdo NM is also shown as it provides an example where the phase of the DV is offset and the amplitude is small compared to all of the other NMs.

Figure 2 shows the same variations as Fig. 1 with the data divided by polarity cycle, as defined in Thomas et al. (2014a), where $A+1$ and $A+2$ are those with positive polarity of the prevailing solar polar field and $A-1$ and $A-2$ are those with negative polarity. The $A+$ and $A-$ labelling here represents whether the polarity of the cycle is positive or negative and the number is which cycle occurred first. For example, $A+1$ is Solar Cycle 21 and $A+2$ is Solar Cycle 23. The percentage change in NM count rates is calculated in the same manner as for Fig. 1. The horizontal dashed line shows where the percentage change in NM from the average is zero. The $y$ axis scales are the same for each NM apart from McMurdo, which has a much smaller DV. Note that the mean variations for each cycle is less than the $0.5 \%$ quoted above, which is due to all of the data being present, including times when no DV was present or when it may have changed phase due to the presence of modulating solar wind transients.

The first thing to note from Fig. 2, is that, generally, the $A>0$ cycles (in blue) peak before than the $A<0$ cycles. This is particularly obvious for Hermanus NM. The amplitude of the NM count rate varies despite the percentage values having been taken from cycle to cycle. This is seen clearly for Alma-Ata NM which varies from a total amplitude of approximately $0.3 \%$ for cycle $A+1$ to $0.6 \%$ for cycle $A-2$. For all NMs, with the exception of Newark, cycle $A-1$ has the large amplitude of the DV, but otherwise there seem to be no cycle-to-cycle trends between the successive amplitudes of the cycles.

To investigate the difference in peaks that is apparent between the $A<0$ and $A>0$ polarity cycles, we now include 

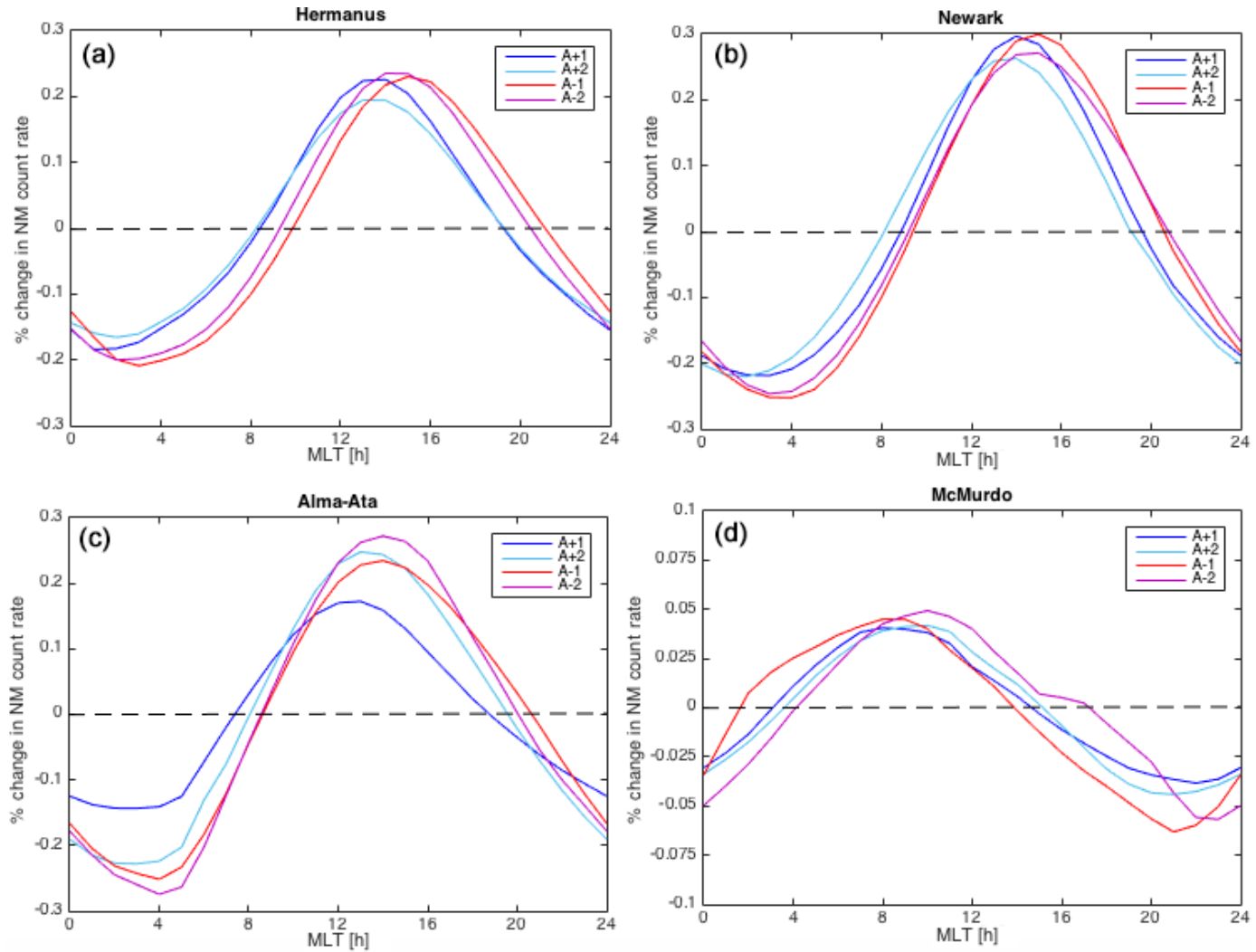

Figure 2. Mean diurnal variations of neutron monitor count rates from Hermanus, South Africa (a), Newark, USA (b), Alma-Ata, Kazakhstan (c), and McMurdo, Antarctica (d). The data are split into four consecutive polarity cycles (see Thomas et al., 2014a), where $A<0$ cycles are shown in red/pink, denoted $A-1$ and $A-2$, and $A>0$ cycles are shown in blue/light blue, denoted $A+1$ and $A+2$.

all NM data available during $A<0$ together into one mean DV and do the same for $A>0$ cycles to compare differences in the phase between the two polarity cycles. Comparing this analysis, shown in Fig. 3, to the polarity cycles shown in Fig. 2, we can look for systematic changes between solar polar polarities.

In Fig. 3, we now take the mean of both the $A<0$ cycles and the $A>0$ cycles to compare the average phase difference in the DV between the two polarity cycles. The data are then split into the mean NM count rates during $A<0$ polarity cycles (red) and those during $A>0$ cycles (blue). The shaded regions of the respective colours represent the standard error on the mean of all data for that particular MLT. The same four NMs are shown for an easy comparison with Fig. 2.

There is a delay of $1-2 \mathrm{~h}$ in the times of the maximum and minimum in the DV between $A>0$ (blue) and $A<0$ (red) cycles for all three lower-latitude stations (Hermanus, Newark and Alma-Ata) shown in Fig. 2. Newark and McMurdo also exhibit consistent patterns in the amplitude of their DVs between the mean $A>0$ and $A<0$ variations. However, Hermanus and Alma-Ata show an offset in the average DV between the two polarity cycles.

However, not all NMs show an observed phase shift. One example is from McMurdo, shown in the lower right of
Fig. 2. Here, the difference in the DV phase between the two polarities is not present, and certainly is within the error on the mean for most times during the cycle. The $A<0$ and $A>0$ DVs peak at a similar time, although the $A<0$ cycles reach a minimum a little later than the $A>0$ cycles. There is a slight change in amplitude, with $A<0$ cycles reaching lower values than the $A<0$ cycles, although this amplitude change appears smaller that than of Alma-Ata. Although changes in the phase of the DVs between polarity cycles have been noted (Bieber and Chen, 1991), the fact that some NMs show different behaviour is intriguing and has not been reported previously. McMurdo NM, for example, displays different behaviour which has an unusual shift in the direction of the maximum GCR flux and a relatively narrow range of ADs at any given time.

To check that the observed change of phase in the DV between polarity cycles is not due to any changes in the heliospheric magnetic field (HMF), we performed the same analysis on the near-Earth HMF magnitude and variation (as calculated in Thomas et al., 2015, using changes in the magnetic field vectors; not shown). This is accessed from the OMNI2 dataset, comprised of data from L1, such as the ACE and WIND spacecraft (King and Papitashvili, 2005). The HMF strength is a key heliospheric parameter to the modulation of 

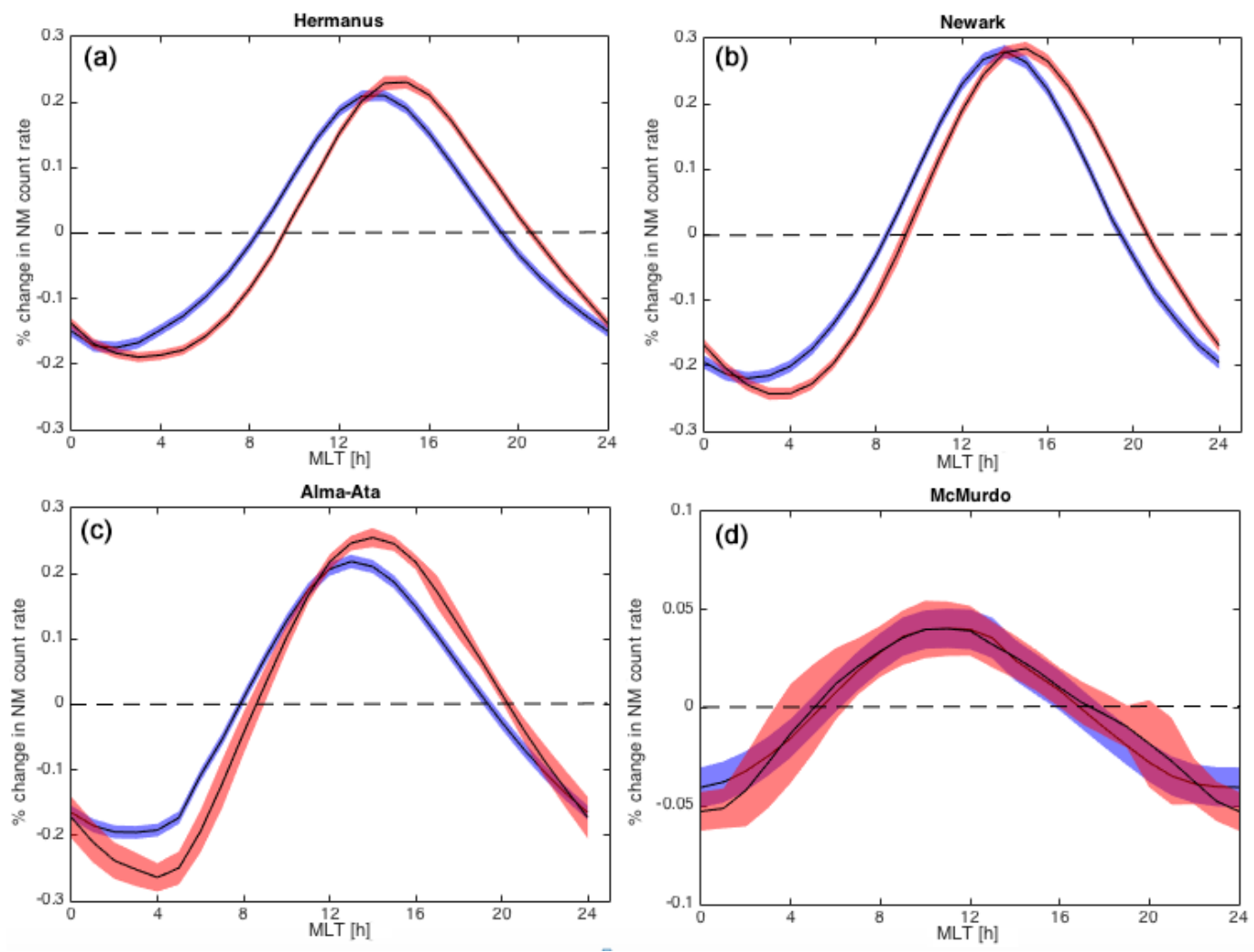

Figure 3. Mean diurnal variations of neutron monitor count rates from Hermanus, South Africa (a), Newark, USA (b), Alma-Ata, Kazakhstan (c), and McMurdo, Antarctica (d). The data are split by the dominant solar polarity, where $A<0$ cycles are shown in red and $A>0$ cycles are shown in blue.

GCRs as they are highly modulated by enhanced and variable magnetic fields via drifts and diffusion processes (Jokipii et al., 1977). We found no statistical changes in the HMF strength, as expected, throughout the average day.

To further remove the effects of solar wind compression from our analysis, and to remove DV arising from DV trains following coronal mass ejections or corotating interaction regions, we locate all times in the HMF data where the HMF strength has surpassed $10 \mathrm{nT}$. This limit, although somewhat arbitrary, is typically only surpassed when there is a moderate-to-large solar wind transient in near-Earth space. For every hourly HMF strength value surpassing this limit, we remove the following 3 days' worth of data from our analysis, when we would expect the largest DVs following such an event. To remove the full recovery phase from a solar wind transient would involve removing at least a week of data each time. As CIRs occur, on average, every 9-10 days (Thomas et al., 2014b), then this would remove a large amount of the data. DV trains also only last this long for the very extreme cases, of which there have not been many of during this time.

Figure 4 shows the same as Fig. 3 but with solar wind transients of a greater than $10 \mathrm{nT}$ HMF magnitude, and with 3 days of data following these transients removed. The first thing to note here is that removing the solar wind transients, and the 3 days' worth of data following, does not remove the difference in the phase of the DV between polarity cycles. Each of Hermanus, Newark and Alma-Ata still have a lag in the DV phase during $A<0$ polarity cycles, compared to $A>0$ cycles. The difference in phase is also somewhat more pronounced for these NMs. Newark, for example, shows that the DV peaks an approximately additional half an hour to an hour later in $A<0$ cycles compared to $A>0$ cycles.

When removing solar wind transients and associated DV trains, the amplitudes, as well as the phase, of the DV between the two polarity cycles do vary for each NM. Although there are more incidences of DV trains at solar maximum, there was a similar amount of DV trains in each cycle resulting in an even spread of removed data from each solar cycle. For Hermanus, the $A<0$ variation has a greater amplitude by approximately $0.1 \%$ than the $A>0$ case. The $A<0$ variation also has a greater amplitude for Newark by approximately $0.05 \%$, Alma-Ata by $0.2 \%$ and McMurdo, by $0.02 \%$. For each case, the difference in amplitude between polarity cycles was much smaller with the solar wind transients included. This can be explained as due to the larger-amplitude DV trains being removed which themselves should not change with heliospheric polarity. The result of this is that the change due to the heliospheric polarity re- 

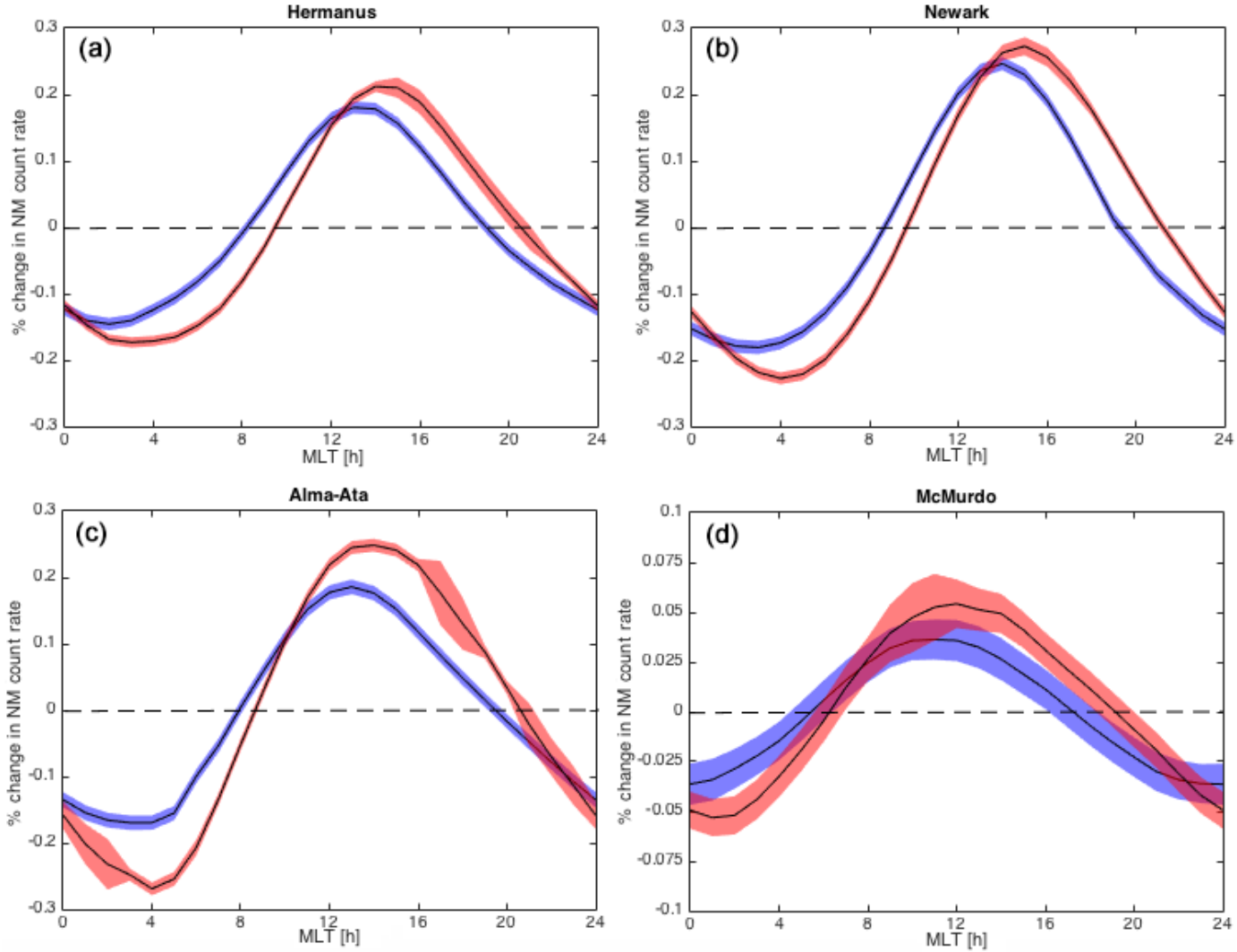

Figure 4. Mean diurnal variations of neutron monitor count rates from Hermanus, South Africa (a), Newark, USA (b), Alma-Ata, Kazakhstan (c), and McMurdo, Antarctica (d). All times where the near-Earth heliospheric magnetic field strength has surpassed $10 \mathrm{nT}$ have been removed, including 3 days of data afterwards to remove DV trains following solar wind transients. The data are split by the dominant solar polarity where $A<0$ cycles are shown in red and $A>0$ cycles are shown in blue.

versal can be observed by itself and is not clouded by the larger-amplitude DV trains. Such a change in amplitude due to heliospheric polarity has not been reported previously but is likely to be caused by the different primary drift directions of GCRs through the heliosphere between the Sun's polarity states.

\section{Latitudinal dependence of phase change}

We now investigate how these changes with heliospheric polarity vary with each NM's geomagnetic cut-off rigidity. The variation in the local magnetic latitude and longitude at each NM site, caused by variations in the structure of the geomagnetic field, can be determined using the International Geomagnetic Reference Field (IGRF; Thébault et al., 2015) model. The difference in the geomagnetic field at the locations of each NM between their start and end dates can then be used as errors on each NM's cut-off rigidity. The largest uncertainties in cut-off rigidity during this period are seen for Newark NM, located in the northeastern USA. This is because the magnetic north pole was located near to Greenland and northeastern Canada at the start of the NM's operation but has since moved considerably towards the geographic north pole in the opposite direction to Newark.

To compare with the latitude of the station, we define a value $\tau$ for each NM, defined as the time of the maximum or minimum in the DV of NM count rates. Therefore the difference between the polarity cycles, $\mathrm{d} \tau$, is defined as $\mathrm{d} \tau=\tau_{A<0}-\tau_{A>0}$. $\mathrm{d} \tau$ is then compared to the geomagnetic cut-off rigidity corresponding to each station, all shown in Table 1. The time shift of the maximum in the DV is labelled $\mathrm{d} \tau_{\max }$, whereas the minimum is labelled $\mathrm{d} \tau_{\min }$. To calculate each $\mathrm{d} \tau$ value, the annual mean DV is found for all NMs listed in Table 1. Each mean was then displayed graphically similarly to Fig. 3, and the maximum and minimum times are read off by clicking on the correct location on the graph and recording the values. Errors were calculated based on the time that the mean count rates plus their standard error fell below the peak mean value. Therefore, the error on $\mathrm{d} \tau$ is greatest when the width of the DV peak and/or trough are narrowest and the standard error on the mean variation is smallest. The calculated $d \tau$ values are plotted against the NM geomagnetic cut-off rigidities in Fig. 5. 


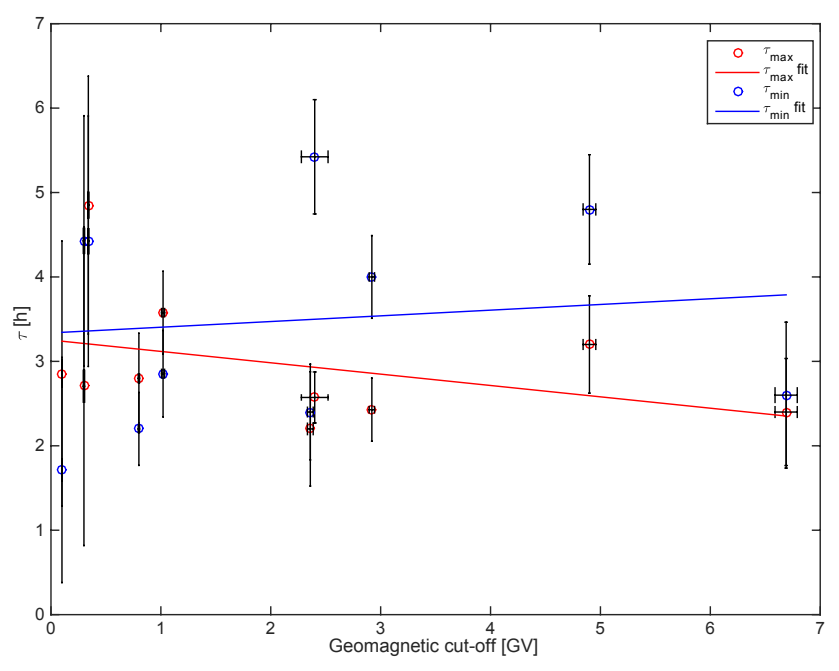

Figure 5. The difference in maximum/minimum time of the DV in neutron monitor count rates between polarity cycles, $\mathrm{d} \tau$, as a function of the neutron monitor's geomagnetic cut-off rigidity, where $\mathrm{d} \tau=\tau_{A<0}-\tau_{A>0}$. The NMs shown (from left to right) are: South Pole $\left(R_{C}=0.1 \mathrm{GV}\right)$, McMurdo $\left(R_{C}=0.3 \mathrm{GV}\right)$, Thule $\left(R_{C}=0.3 \mathrm{GV}\right)$, Oulu $\left(R_{C}=0.8 \mathrm{GV}\right)$, Deep River $\left(R_{C}=1.02 \mathrm{GV}\right)$, Kiel $\left(R_{C}=2.36 \mathrm{GV}\right)$, Newark $\left(R_{C}=2.4 \mathrm{GV}\right)$, Climax $\left(R_{C}=2.92 \mathrm{GV}\right)$, Hermanus $\left(R_{C}=4.9 \mathrm{GV}\right)$ and Alma Ata $\left(R_{C}=6.69 \mathrm{GV}\right)$. The data are shown by the hexagons with $x$ and $y$ errors shown by the black crosses. Errors on the geomagnetic cut-off rigidities are calculated from the local variations in the geomagnetic field over the time span of the NM data, whereas the errors on $\mathrm{d} \tau$ are calculated from the time taken for NM count rates plus their standard error to fall below the peak mean NM count rate value. The lines are best fits of the data, which are plotted just for reference. Thule is shifted slightly to the right to appear slightly apart from McMurdo.

Figure 5 shows the geomagnetic cut-off rigidity of each NM used in the study against $\mathrm{d} \tau$. The $x$-axis error bars are from the variation in the magnetic latitude over time, as described above, and the $y$-axis errors are the time taken for the mean variation plus the standard error to exceed the mean NM count rate peak value. In most cases, $\mathrm{d} \tau_{\min }$ is longer than $\mathrm{d} \tau_{\max }$ indicating that the time of the minimum in the DV shifts more in time than the maximum. For half of the $\mathrm{d} \tau_{\min }$ values, we observe time differences between $A<0$ and $A>0$ peak times of $>4 \mathrm{~h}$. However, $\mathrm{d} \tau_{\max }$ is longer than $\mathrm{d} \tau_{\min }$ for Oulu, Deep River, Thule and South Pole. These results indicate that the change in DV between polarity cycles in not a change in phase, but rather an asymmetric change in the GCR flux arriving at any given NM.

Best-fit lines are calculated and shown on Fig. 5 for reference as they are not statistically verified correlations. Correlation coefficients for $\mathrm{d} \tau_{\max }$ and $\mathrm{d} \tau_{\min }$ were calculated giving values of 0.5 and 0.1 . There is clearly no trend in the $\tau_{\min }$ data with geomagnetic cut-off rigidity. However, the $\mathrm{d} \tau_{\max }$ values do hint at having a slight tendency to decrease with

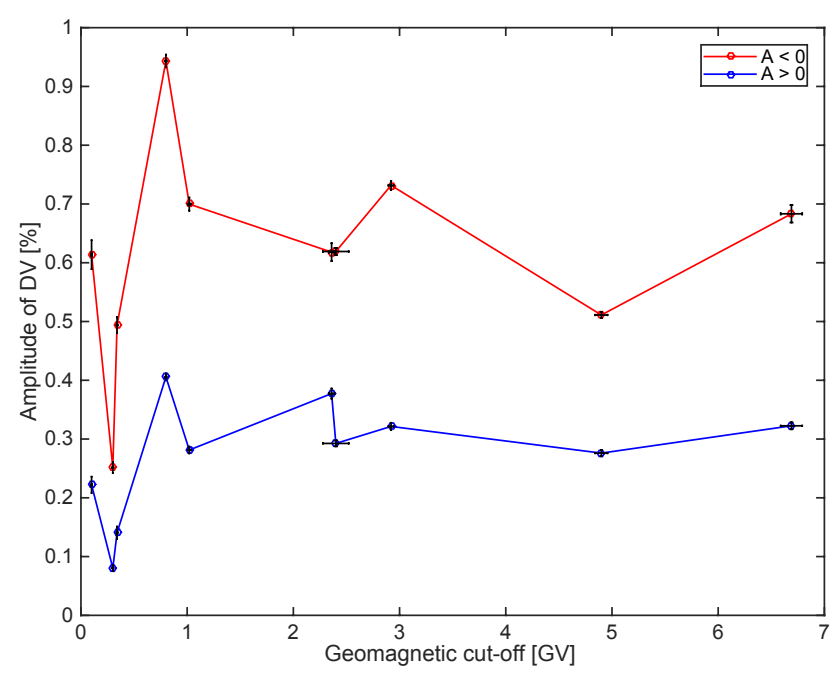

Figure 6. The amplitude of the diurnal variation at each neutron monitor as a function of the neutron monitor's cut-off rigidity. The red line is for $A<0$ polarity cycles and the blue is for $A>0$ cycles. The NMs shown (from left to right) are: South Pole $\left(R_{C}=0.1 \mathrm{GV}\right)$, McMurdo $\left(R_{C}=0.3 \mathrm{GV}\right)$, Thule $\left(R_{C}=0.3 \mathrm{GV}\right)$, Oulu $\quad\left(R_{C}=0.8 \mathrm{GV}\right)$, Deep River $\left(R_{C}=1.02 \mathrm{GV}\right)$, Kiel $\left(R_{C}=2.36 \mathrm{GV}\right)$, Newark $\left(R_{C}=2.4 \mathrm{GV}\right), \operatorname{Climax}\left(R_{C}=2.92 \mathrm{GV}\right)$, Hermanus $\left(R_{C}=4.9 \mathrm{GV}\right)$ and Alma Ata $\left(R_{C}=6.69 \mathrm{GV}\right)$. The data are shown by the circles with $x$ and $y$ errors shown by the black crosses. Errors on the geomagnetic cut-off rigidities are calculated from the local variations in the geomagnetic field over the time span of the neutron monitor data, whereas the error on the amplitude is the average standard error between the peak and trough of the DV.

increasing NM cut-offs meaning that the time difference in the maximum of the DV changes slightly more at higher latitudes. The $\mathrm{d} \tau_{\max }$ best fit, however, does seem to be influenced by the timing of the maximum between polarity cycles at the McMurdo NM, which has been shown to be unusual with respect to the other NMs.

Figure 6 shows the amplitude of the DV for each polarity cycle as a function of the geomagnetic cut-off rigidity of each NM for $A<0$ polarity cycles (red) and $A>0$ cycles (blue). The error bars in the $x$ direction are the same as for Fig. 5, whereas the $y$-direction error bars are the mean of the standard error of the maximum and minimum NM count rate values. The first thing to note from Fig. 6 is that the shape of the variation with magnetic latitude is remarkably similar for $A<0$ and $A>0$ polarity cycles. In both cases, Oulu has the greatest amplitude of the DV, whereas McMurdo has the smallest, as was also seen in Fig. 1. The large difference in the amplitude between the high values for Oulu and South Pole NMs compared to the low values for McMurdo and Thule is initially somewhat surprising giving the similar geomagnetic cut-off rigidities of the stations. As only highrigidity particles can access NMs of high geomagnetic cutoff rigidities, it would be reasonable to expect the amplitude 


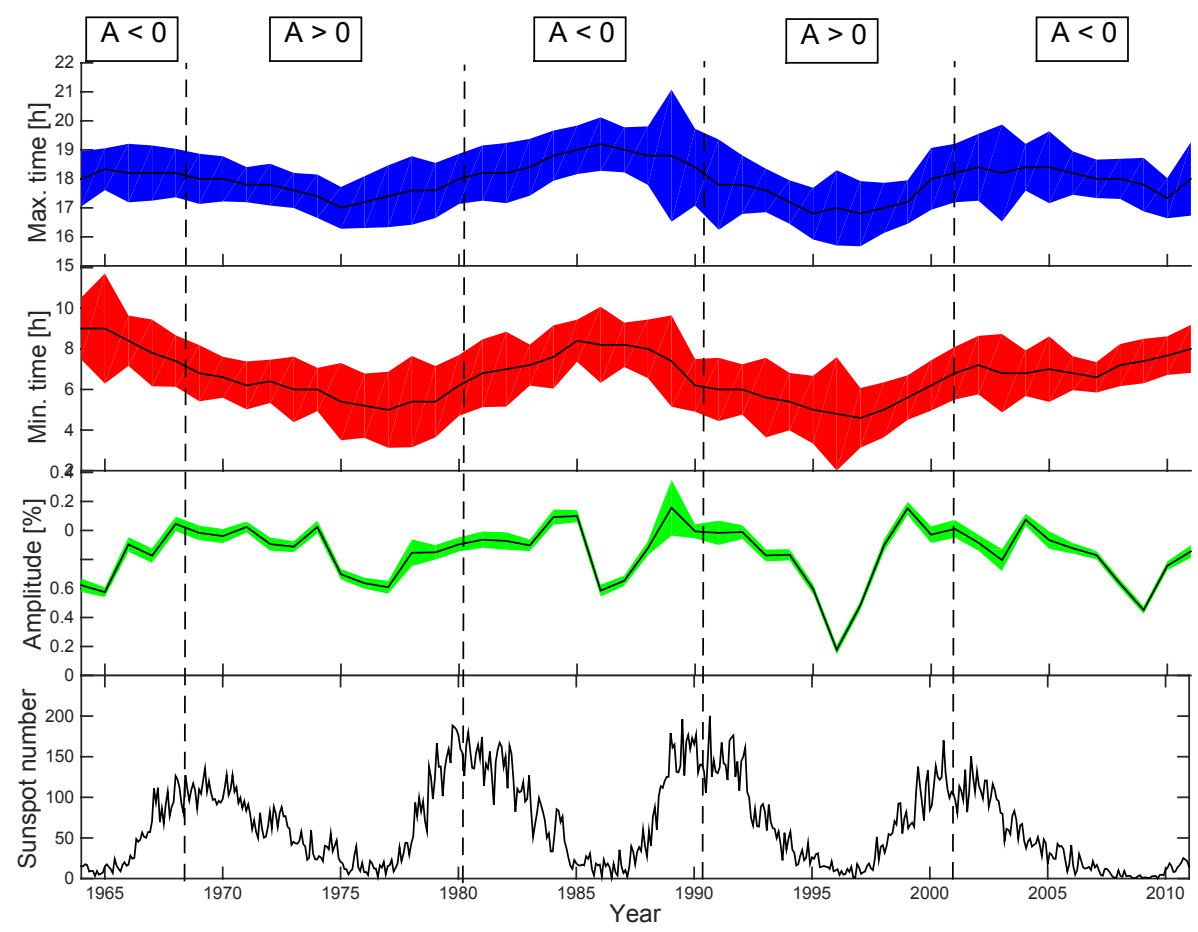

Figure 7. Neutron monitor data is from Newark, USA $\left(37.9^{\circ} \mathrm{N}, 75.7^{\circ} \mathrm{W}\right)$. Mean times of day of (from top) the maximum in the diurnal variation of neutron monitor counts, the minimum, the mean amplitudes of the variation and the international sunspot number. Shaded regions are the error calculated for individual years as the times. The computation of uncertainties is described in the text. The vertical dashed lines are times of polarity reversal estimate by Thomas et al. (2014a) and the solar magnetic polarity of each cycle is labelled at the top.

of the DV to be smaller at these stations, compared to the polar NMs. However, no relation is observed with the NM's geomagnetic cut-off rigidity. This is therefore due to the height and direction of each NM's AD. McMurdo's AD is unusual with respect to the other NMs and thus it is perhaps unsurprising that it shows an unusually low amplitude.

Figure 6 also gives a good illustration of the changing amplitude, as well as phase, of the DV with solar polar polarity. The mean amplitude of the DV is much greater during $A<0$ cycles than during $A>0$ cycles for all NMs in the study, frequently by a factor of 2 or more, which cannot be accounted for by the spread of amplitude values going into the mean. It seems that this change could only be due to the different magnetic field configuration in the heliosphere between polarity cycles imposing different drift pattern on the GCRs. However, the latest $A<0$ polarity cycle has been associated with weak HMF strengths and high NM count rates which may have an influence on DV amplitude. However, it is unlikely to account for the factor of 2 difference between the polarities.

\section{Eleven- and 22-year patterns in diurnal neutron monitor data}

We now compute the mean DV for each year to investigate how the DV changes on a yearly basis, and whether the change in maximum and minimum times in the DV and its amplitude are gradual, or more of a sudden reversal in regime. Once an average DV profile is deduced for each year, it is then smoothed using a moving average filter. The time of the maxima and minima of the resulting smoothed curves are then deduced, along with the amplitude of the DV (i.e. the difference between the maximum and minimum values). The times of the maxima and minima, as well as the amplitude of the DV can then be plotted against time to see how each varies with time, as is shown in Figs. 7-9.

The MLTs of the maxima and minima for the Newark NM are shown in Fig. 7. The maxima (blue), minima (red) and amplitude (green) of the mean DV for each year are shown in the top three panels. These are compared to the international sunspot record (black) used as a proxy for solar activity. The uncertainty on the mean for the times of maximum (minimum) in the DV are calculated as the average time for the mean NM count rate plus (minus) the standard error to fall below (rise above) the maximum (minimum) mean count rate. The amplitude is calculated as the difference between the maxima and minima of the mean DV for each year. The 


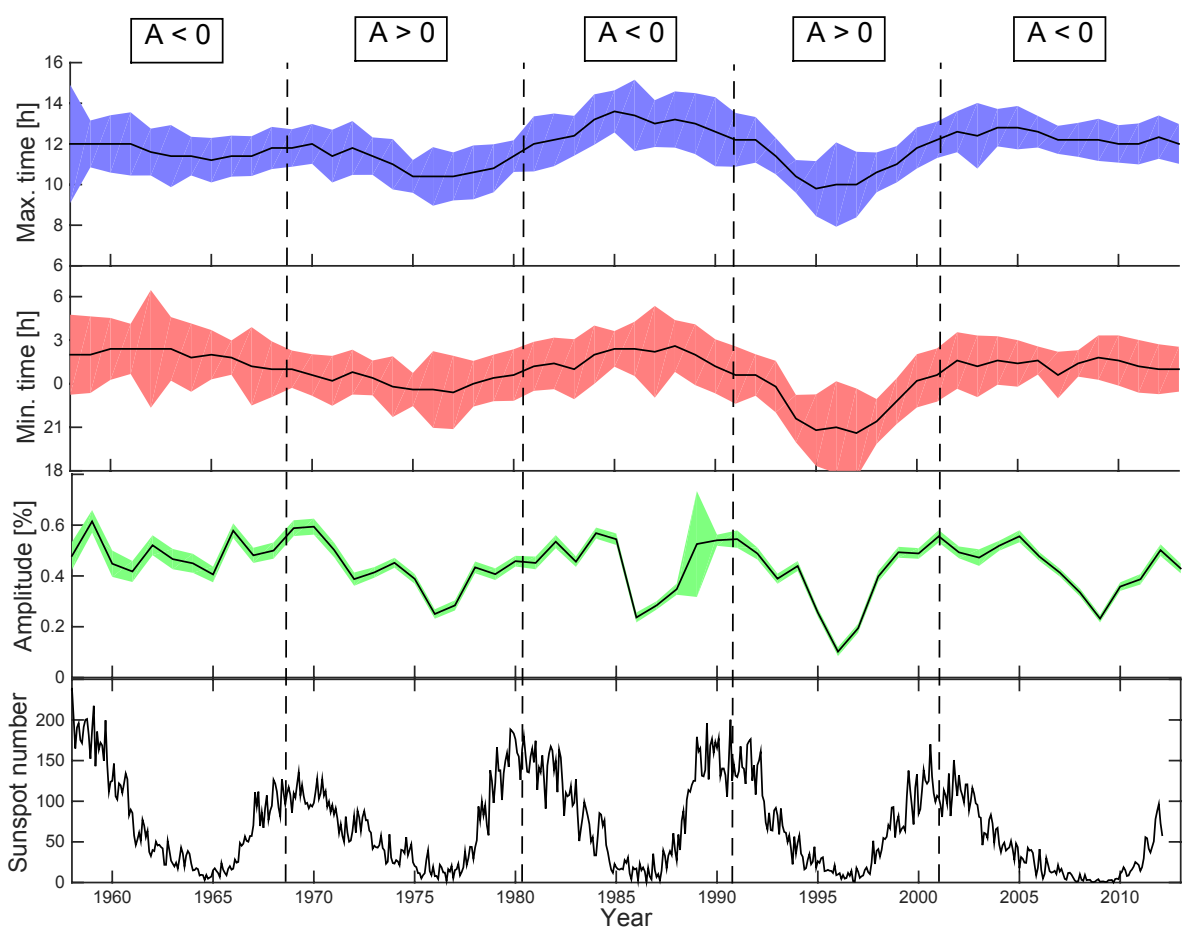

Figure 8. Neutron monitor data from Hermanus, South Africa $\left(34.4^{\circ} \mathrm{S}, 19.2^{\circ} \mathrm{E}\right)$. Mean times of day of (from top) the maximum in the diurnal variation of neutron monitor counts, the minimum, the mean amplitudes of the variation and the international sunspot number. Shaded regions are the error calculated for individual years as the times. The computation of uncertainties is described in the text. The vertical dashed lines are times of polarity reversal estimate by Thomas et al. (2014a) and the solar magnetic polarity of each cycle is labelled at the top.

error bars on the amplitude represents the error on the mean between the maximum and minimum count rates. Finally, the vertical dashed lines are the best-fit times of polarity reversal calculated by Thomas et al. (2014a).

Both the maxima and the minima times vary in a 22-year cycle, related to the phase of the DV (Bieber and Chen, 1991). Comparing the top two panels of Fig. 5, variations in the maxima and minima are in phase such that the whole DV is shifted to earlier MLT by up to approximately $2 \mathrm{~h}$ during $A<0$ polarity cycles compared to $A>0$ cycles. There is some evidence that the solar minimum of the late 1960s has a much larger shift towards a later minimum in the DV than a later maximum. The latest hour of the maximum/minimum in the DVs had not been reached as of 2012, which could be symptomatic of the unusual behaviour of the Sun during this period (e.g. Barnard et al., 2011; Lockwood et al., 2012). Furthermore, the change in the maximum and minimum times of the yearly mean DV is clearly a gradual one between $A<0$ and $A>0$ polarity cycles as the difference in times cycle with the 22-year variation in a sinusoidal-like manner.

The third panel of Fig. 7 shows time series of the amplitude of the DV. In agreement with previous studies, the amplitude shows an 11-year variation. The largest amplitudes are found at solar maximum and are sustained into the declining phase of the solar cycle for all four cycles. This is likely due to there being more modulating structures present during these times with more frequent coronal mass ejections (Webb and Howard, 1994), stream-stream interaction regions with large latitudinal extent (Rouillard and Lockwood, 2007), or greater magnetic flux in the heliosphere (Lockwood et al., 2009), which increases particle drift effects and diffusion, respectively, reducing the local GCR flux (Jokipii and Kopriva, 1979). There is no evidence from Fig. 7 of a 22 -year variation in the amplitude, or indeed a solar cycle variation in the diurnal maximum and minimum times of day as was reported by $\mathrm{Oh}$ et al. (2010). However, Fig. 4 does show there is a difference between polarity cycles. As the amplitude varies in an 11-year cycle, the difference shown in Fig. 4 for Newark seems quite dependent on the small amplitudes seen during the $A>0$ cycle in the 1990 s, which could also explain why our results are different to those of Oh et al. (2010) in Fig. 7.

Figures 8 and 9 show the same as Fig. 7 but for the neutron monitors at Hermanus, South Africa, and Alma-Ata, Kazakhstan, respectively. For Hermanus (Fig. 8), the time of the DV maximum varies from 10:00 to 14:00 UTC, whereas the minimum time varies from 20:00 to 03:00 UTC. The period 1993-1997 is particularly notable as the timings of the maximum and minimum in the DV both move much earlier than is observed at any other time in the data. We also observe a suppressed amplitude in the DV at this time, similarly to that seen in Fig. 7, which coincided with the minimum of the 11-year solar cycle. 


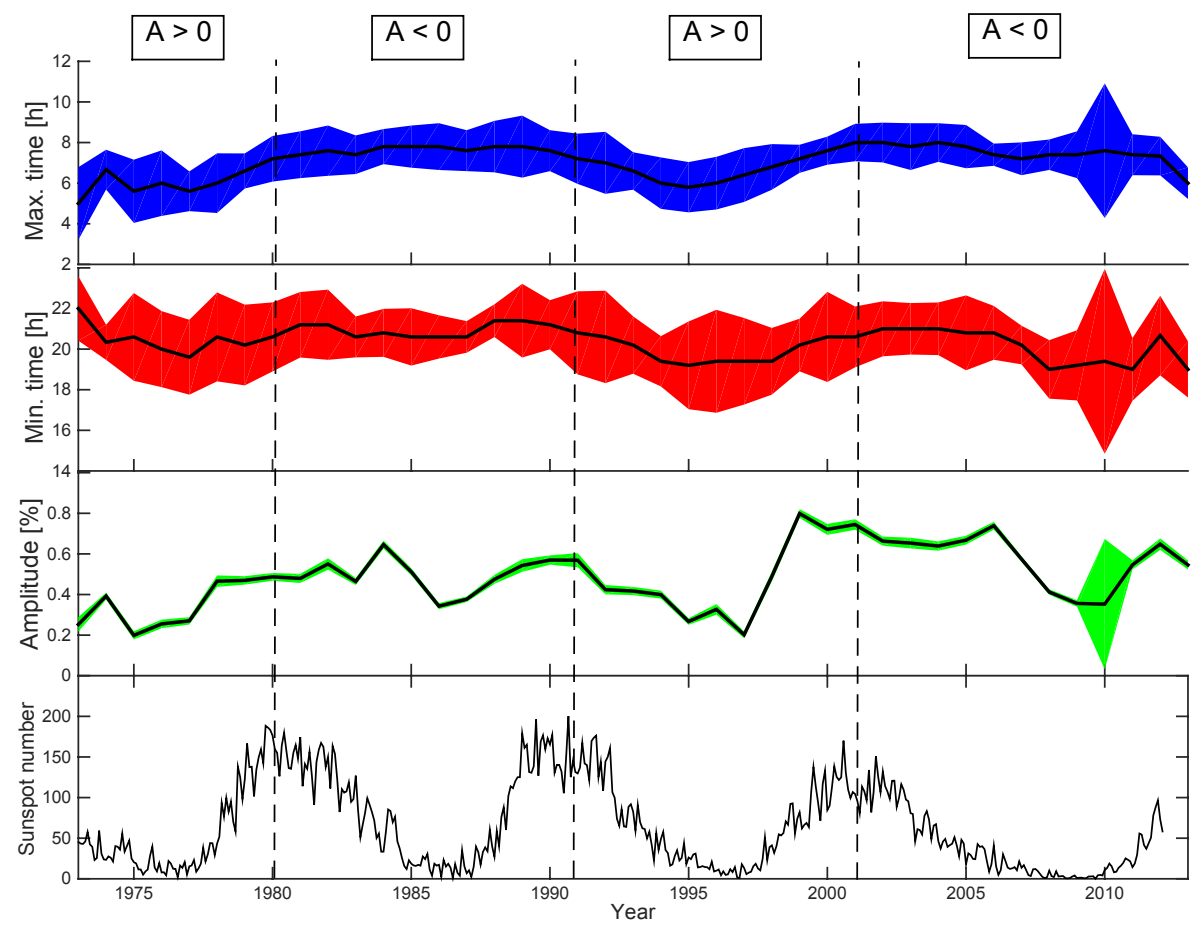

Figure 9. Neutron monitor data are from Alma-Ata, Kazakhstan $\left(43.3^{\circ} \mathrm{N}, 76.9^{\circ} \mathrm{E}\right)$. Mean times of day of (from top) the maximum in the diurnal variation of neutron monitor counts, the minimum, the mean amplitudes of the variation and the international sunspot number. Shaded regions are the error calculated for individual years as the times. The computation of uncertainties is described in the text. The vertical dashed lines are times of polarity reversal estimate by Thomas et al. (2014a) and the solar magnetic polarity of each cycle is labelled at the top.

For Alma-Ata (Fig. 9), the time of DV varies from 05:00 to 08:00 UTC for the maximum time and from 18:00 to 22:00 UTC for the minimum time. The 22-year variation has a slightly greater amplitude for the times of maxima than the minima with a change to earlier peaks at the solar minima in the late 1970s and 1990s. The solar cycle variation in the amplitude of the DV is not consistent with consecutive cycles. For example, the most recent cycle is very clear with an amplitude of the mean variation of approximately $0.8 \%$, but there is very little change in the amplitude for the previous cycle. The 22-year variation is again evident for both the timings of the maxima and minima for Hermanus and Alma-Ata. Hermanus has a much larger cycle in the peak time between 1975 and 1997 but has a much less clear change for the previous cycle, especially for the maximum times. Furthermore, there is no decrease in the amplitude for the 1964-1965 solar minimum. The amplitude of the DV is much greater between 1997 and 2007, either side of the polarity reversal of 2001. In this case, the enhanced DV throughout the recent weak solar cycle could have been the cause of the amplitude difference between $A<0$ and $A>0$ polarity cycles.

\section{Conclusions}

We have examined changes in the diurnal variation (DV) of cosmic rays with the Sun's dominant solar polar polarity and with the geomagnetic cut-off rigidity of each neutron monitor station. Mean neutron monitor count rates for the majority of the stations show a sinusoidal-type curve with a peak at 14:00-17:00 MLT and trough between 03:00 and 06:00 MLT, in agreement with the previous literature (e.g. Pomerantz and Duggal, 1970). McMurdo, Antarctica, is a notable exception from this rule, with a peak at around 10:00 MLT and trough at 00:00 MLT. This difference is likely related to the low amplitude of the variation, which in turn is due to McMurdo's location at a very high latitude. The asymptotic directions of most NMs are shifted eastwards by approximately $60^{\circ}$, whereas McMurdo's shifts differently resulting in a different phase in the DV.

We observe a difference in the phase and amplitude of the DV in neutron monitor count rates with the Sun's magnetic polarity. The timing varies in a 22 -year cycle with maxima and minima latest in the day at solar minimum in $A<0$ cycles and earliest at solar minimum during $A>0$ cycles. These results are shown to be in agreement with Forbush (1969) and Bieber and Chen (1991), who also noted a 22year variation in the mean DV. For all neutron monitors used in this study, the time of day of the maximum and minimum 
in the DV vary by $1-6 \mathrm{~h}$ between $A<0$ and $A>0$, heliospheric magnetic polarities.

The amplitude and time difference between polarity cycles is different depending on the neutron monitor in question. Alma-Ata-B, for example, shows a large difference in phase and amplitude, whereas McMurdo displays a much smaller variation. However, the times of the maximum and minimum in the DV vary differently with the change in magnetic polarity. This implies that the variation in the timing of the DV, and hence the sense of anisotropy of GCRs reaching Earth, should not be treated as a phase change but more an nonsymmetric change in GCR anisotropy.

The fact that the whole DV shifts later in the day during $A<0$ polarity cycles compared to $A>0$ cycles suggests a variation in the primary directions that GCR are drifting through the inner heliosphere to Earth. There are also local sensitivities in the locations of the neutron monitors to the changes in GCR anisotropy with time observed at most locations. In general, however, the peak in GCR flux occurs approximately $2 \mathrm{~h}$ later, corresponding to an approximately $30^{\circ}$ difference in the preferred direction of anisotropy during $A<0$ cycles compared to $A>0$ cycles.

When removing solar wind transient effects from the data, the difference in the diurnal variation between heliospheric polarities becomes more pronounced. There is a large difference between the times of maxima and minima in the DV and also a greater amplitude variation between the polarities. To completely remove all diurnal trains caused by solar wind transients, at least 7 days' worth of data would be removed after a transient crossed Earth, rather than the 3 we have taken, but this would remove a far too large proportion of the data.

Data availability. The data used in this study are all freely accessible from existing sources. The neutron monitor data can be found on the Neutron Monitor Database website (http://nmdb.eu), the Bartol neutron monitors' webpage (http://neutronm.bartol.udel.edu), and the Izmuran neutron monitor interface (http://cr0.izmiran.ru/ common/links.htm) and, for the case of the Hermanus neutron monitor, can be access freely from North-West University's webpage (http://natural-sciences.nwu.ac.za/neutron-monitor-data). The International Sunspot Number is freely accessed from https://www. ngdc.noaa.gov/stp/solar/ssndata.html. Solar wind transients were identified from the OMNI2 data accessed from https://omniweb. gsfc.nasa.gov/html/ow_data.html.

Competing interests. The authors declare that they have no conflict of interest.

Acknowledgements. We are grateful for the use of the neutron monitor database accessed from http://www.nmdb.eu and the Izmuran database, as well as each of the neutron monitor's operators. The operators we would like to thank include the Bartol Institute, who operate the McMurdo, Thule, Newark and South Pole neutron monitors; North-West University for the Hermanus data; the University of Oulu; the University of Kiel; and the operators of the Climax, Deep River and Alma-Ata-B neutron monitors. The work of SRT was supported by the STFC. M. Owens and M. Lockwood are supported under STFC grant no. ST/M000885/1. We are also grateful to NASA's Goddard Space Flight Center for the use of the OMNI2 solar wind data accessed from https://omniweb.gsfc.nasa.gov/html/ ow_data.html.

The topical editor, Manuela Temmer, thanks Konstantin Herbst and one anonymous referee for help in evaluating this paper.

\section{References}

Ahluwalia, H. S.: Cosmic Ray Transverse Gradient for a Hale Cycle, J. Geophys. Res., 99, 23515-23521, 1994.

Barnard, L., Lockwood, M., Hapgood, M. A., Owens, M. J., Davis, C. J., and Steinhilber, F.: Predicting space climate change, Geophys. Res. Lett., 38, L16103, https://doi.org/10.1029/2011GL048489, 2011.

Bazilevskaya, G. A., Usoskin, I. G., Flueckiger, E. O., Harrison, R. G., Desorgher, L., Buetikofer, R., Krainev, M. B., Makhmutov, V. S., Stozhkov, Y. I., Svirzkevskaya, A. K., Svirzhevskaya, N. S., and Kovaltsov, G. A.: Cosmic Ray Induced Ion Production in the Atmosphere, Space Sci. Rev., 137, 149-173, 2008.

Bercovitch, M. and Robertson, B. C.: Meteorological factors affecting the counting rate of neutron monitors, Proceedings of the 9th International Cosmic Ray Conference, London, 1, 489-491, 1965.

Bieber, J. W. and Chen, J.: Cosmic Ray Diurnal Anisotropy, 19361988: Implications to Drift and Modulation Theories, Astrophys. J., 372, 301-313, 1991.

Bieber, J. W. and Evenson, P.: CME geometry in relation to cosmic ray anisotropy, Geophys. Res. Lett., 25, 2955-2958, 1998.

Cordaro, E. G., Galvez, D., and Laroze, D.: Observation of intensity of cosmic rays and daily magnetic shifts near meridian $70^{\circ}$ in the South America, J. Atmos. Sol.-Terr. Phy., 142, 72-82, 2016.

Dumbovic, M., Vrsnak, B., and Calogovic, J.: Forbush Decrease Prediction Based on Remote Solar Observations, Sol. Phys., 291, 285-302, https://doi.org/10.1007/s11207-015-0819-4, 2015.

Dungey, J. W.: Interplanetary Magnetic Field and the Auroral Zones, Phys. Rev. Lett.,6, 47-48, 1961.

Flückiger, E. O., Smart, D. F., Shea, M. A., and Gentile, L. C.: On the correlation between asymptotic directions of cosmic ray particles and cutoff rigidities in the evolving geomagnetic field, J. Geophys. Res., 92, 2551-2554, 1985.

Forbush, S. E.: On the effects in cosmic-ray intensity observed during the recent magnetic storm, Phys. Rev., 51, 1108-1109, 1937.

Forbush, S. E. and Venkatesan, D.: Diurnal Variation in CosmicRay Intensity, 1937-1959, at Cheltenham (Fredericksburg), Huancayo, and Christchurch, J. Geophys. Res., 65, 2213, https://doi.org/10.1029/JZ065i008p02213, 1960.

Forbush, S. E.: Variation with a period of two solar cycles in the cosmic-ray diurnal anisotropy and the superposed variations correlated with magnetic activity, J. Geophys. Res., 74, 3451, https://doi.org/10.1029/JA074i014p03451, 1969. 
Forbush, S. E.: Cosmic ray diurnal anisotropy 1937-1972, J. Geophys. Res., 78, 7933, https://doi.org/10.1029/JA078i034p07933, 1973.

Gil, A., Usoskin, I. G., Kovaltsov, G. A., Mishev, A. L., Corti, C., and Bindi, V.: Can we properly model the neutron monitor count rate?, J. Geophys. Res., 120, 7172-7178, 2015.

Herbst, K., Kopp, A., and Heber, B.: Influence of the terrestrial magnetic field geometry on the cutoff rigidity of cosmic ray particles, Ann. Geophys., 31, 1637-1643, https://doi.org/10.5194/angeo31-1637-2013, 2013.

Jokipii, J. R., Levy, E. H., and Hubbard, W. B.: Effects of particle drift on cosmic-ray transport. I. General properties, application to solar modulation, Astrophys. J., 213, 861-868, 1977.

Jokipii, J. R. and Kopriva, D. A.: Effects of particle drift on the transport of cosmic rays. III - Numerical models of galactic cosmic-ray modulation, Astrophys. J., 234, 384-392, 1979.

King, J. H. and Papitashvili, N. E.: Solar wind spatial scales in and comparisons of hourly wind and ACE plasma and magnetic field data, J. Geophys. Res., 110, A02104, https://doi.org/10.1029/2004JA010649, 2005.

Kobelev, P., Belov, A., Mavromichalaki, E., Gerontidou, M., and Yanke, V.: Variations of Barometric Coefficients of the Neutron Component in the 22-23 Cycles of Solar Activity, Proc. 32nd ICRC, 2011.

Lapointe, S. M. and Rose, D. C.: The effective directional sensitivity of cosmic-ray neutron monitors, Can. J. Phys., 39, 668-676, https://doi.org/10.1139/p61-078, 1961.

Lockwood, M., Rouillard, A. P., and Finch, I. D.: The Rise and Fall of Open Solar Flux During the Current Grand Solar Maximum, Ap. J., 700, 937-944, 2009.

Lockwood, M., Owens, M. J., Barnard, L., Davis, C. J., and Thomas, S. R.: What is the Sun up to?, Astron. Geophys., 53 3.09-3.15, 2012.

Mavromichalaki, H., Papaioannou, A., Plainaki, C., Sarlanis, C., Souvatzoglou, G., Gerontidou, M., Papailiou, M., Eroshenko, E., Belov, A., Yanke, V., Flückiger, E. O., Bütikofer, R., Parisi, M., Storini, M.; Klein, K.-L., Fuller, N., Steigies, C. T., Rother, O. M., Heber, B., Wimmer-Schweingruber, R. F., Kudela, K., Strharsky, I., Langer, R., Usoskin, I., Ibragimov, A., Chilingaryan, A., Hovsepyan, G., Reymers, A., Yeghikyan, A., Kryakunova, O., Dryn, E., Nikolayevskiy, N., Dorman, L., and Pustil'Nik, L.: Applications and usage of the real-time Neutron Monitor Database, Adv. Space Res., 47, 2210-2222, 2011.

Moraal, H., Belov, A., and Clem, J. M.: Design and co-Ordination of Multi-Station International Neutron Monitor Networks, Space Sci. Rev., 93, 285-303, 2000.

Milan, S. E., Gosling, J. S., and Hubert, B.: Relationship between interplanetary parameters and the magnetopause reconnection rate quantified from observations of the expanding polar cap, J. Geophys. Res., 117, A03226, https://doi.org/10.1029/2011JA017082, 2012.

Mishev, A. L., Usoskin, I. G., and Kovaltsov, G. A.: Neutron monitor yield function: New improved computations, J. Geophys. Res., 118, 2783-2788, 2013.

Mishra, R. K. and Mishra, R. A.: Cosmic ray daily variation and solar activity on anomalous days, Rom. J. Phys., 53, 925-932, 2013.
Moraal, H. and Stoker, P. H.: Long-term neutron monitor observations and the 2009 cosmic ray maximum, J. Geophys. Res., 115, A12109, https://doi.org/10.1029/2010JA015413, 2010.

Oh, S. Y., Yi, Y., and Bieber, J. W.: Modulation Cycles of Galactic Cosmic Ray Diurnal Anisotropy Variation, Sol. Phys., 262, 199_ 212, 2010.

Owens, M. and Cargill, P.: Predictions of the arrival time of Coronal Mass Ejections at 1AU: an analysis of the causes of errors, Ann. Geophys., 22, 661-671, https://doi.org/10.5194/angeo-22661-2004, 2004.

Parker, E. N.: Theory of streaming of cosmic rays and the diurnal variation, Planet. Space Sci., 12, 735-749, 1964.

Parker, E. N.: The Passage of Energetically Charged Particles through Interplanetary Space, Planet. Space Sci., 13, 9-49, 1965.

Plainaki, N., Mavromichalaki, H., Belov, A., Eroshenko, E., and Yanke, V.: Neutron monitor asymptotic directions of viewing during the event of 13 December 2006, Adv. Space Res., 43, 518-522, 2009.

Pomerantz, M. A. and Duggal, S. P.: The Cosmic Ray Solar Diurnal Anisotropy, Space Sci. Rev., 12, 75-130, 1970.

Potgieter, M. S.: Solar Modulation of Cosmic Rays, Liv. Rev. Sol. Phys., 10, 66, https://doi.org/10.12942/lrsp-2013-3, 2013.

Rao, U. R., McCracken, K. C., and Venkatesan, D.: Asymptotic Cones of Acceptance and Their Use in the Study of the Daily Variation of Cosmic Radiation, J. Geophys. Res., 68, 345-369, 1963.

Raubenheimer, B. C. and Stoker, P. H.: Various aspects of the attenuation coefficient of a neutron monitor, J. Geophys. Res., 79, 5069-5076, 1974.

Richardson, I. G. and Cane, H. V.: The relationship between recurring cosmic ray depressions and corotating solar wind streams at $<=1$ AU: IMP 8 and Helios 1 and 2 anticoincidence guard rate observations, J. Geophys. Res., 101, 13483-13496, 2010.

Rouillard, A. and Lockwood, M.: Oscillations in the open solar magnetic flux with a period of 1.68 years: imprint on galactic cosmic rays and implications for heliospheric shielding, Ann. Geophys., 22, 4381-4395, https://doi.org/10.5194/angeo22-4381-2004, 2004.

Rouillard, A. P. and Lockwood, M.: The latitudinal effect of corotating interaction regions on galactic cosmic rays, Sol. Phys., 245, 191-206, 2007.

Smart, D. F., Shea, M. A., and McCracken, K. C.: A Study of Vertical Cutoff Rigidities Using Sixth Degree Simulations of the Geomagnetic Field, J. Geophys. Res., 70, 4117-4130, 1965.

Tezari, A. and Mavromichalaki, H.: Diurnal anisotropy of cosmic rays during intensive solar activity for the period 2001-2014, New Astron., 46, 78-84, 2016.

Thébault, E., Finley, C. C., Beggan, C. D., Alken, P., Aubert, J., Barrois, O., Bertrand, F., Bondar, T., Boness, A., Brocco, L., Canet, E., Chambodut, A., Chulliat, A., Coïsson, P., Civet, F., Du, A., Fournier, A., Fratter, I., Gillet, N., Hamilton, B., Hamoudi, M., Hulot, G., Jager, T., Korte, M., Kuang, W., Lalanne, X., Langlais, B., Léger, J.-M., Lesur, V., Lowes, F. J., MacMillan, S., Mandea, M., Manoj, C., Maus, S., Olson, M., Petrov, V., Ridley, V., Rother, M., Sabaka, T. J., Saturnino, D., Schachtscheider, R., Sirol, O., Tangborn, A., Thomson, A., Toffner-Clausen, L., Vigneron, P., Wardinski, I., and Zvereva, T.: International Geomagnetic Reference Field: the 12th generation, Earth Planets and Space, 67, 67-79, 2015. 
Thomas, S. R., Owens, M. J., and Lockwood, M.: The 22-Year Hale Cycle in Cosmic Ray Flux - Evidence for Direct Heliospheric Modulation, Sol. Phys., 289, 407-421, 2014a.

Thomas, S. R., Owens, M. J., and Lockwood, M.: Galactic Cosmic Ray Modulation in the Heliosphere, Astron. Geophys., 55, 2325, 2014b.

Thomas, S. R., Owens, M. J., Lockwood, M., Barnard, L., and Scott, C. J.: Near-Earth Cosmic Ray Decreases Associated with Remote Coronal Mass Ejections, Ap. J., 801, 5, https://doi.org/10.1088/0004-637X/801/1/5, 2015.

Thomas, S. R., Fazakerley, A., Wicks, R., and Green, L.: Evaluating the skill of forecasts of the Near-Earth solar wind using a space weather monitor at L5, Space Weather, in press, 2017.
Usoskin, I. G., Bazilevskaya, G. A., and Kovaltsov, G. A.: Solar modulation parameter for cosmic rays since 1936 reconstructed from ground-based neutron monitors and ionization chambers, J. Geophys. Res., 116, A02104, https://doi.org/10.1029/2010JA016105, 2011.

Vainio, R., Desorgher, L., Heynderickx, D., Storini, M., Flueckiger, E., Horne, R. B., Kovaltsov, G. A., Kudela, K., Laurenza, M., McKenna-Lawlor, S., Rothkaehl, H., and Usoskin, I. G.: Dynamics of the Earth's Particle Radiation Environment, Space Sci. Rev., 147, 187-231, 2009.

Webb, D. F. and Howard, T. A.: The solar cycle variation of coronal mass ejections and the solar wind mass flux, J. Geophys. Res., 99, 4201-4220, 1994. 